Supplement of Hydrol. Earth Syst. Sci., 24, 169-188, 2020

https://doi.org/10.5194/hess-24-169-2020-supplement

(c) Author(s) 2020. This work is distributed under

the Creative Commons Attribution 4.0 License.

(c) (1)

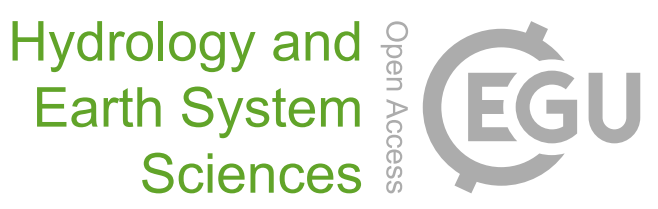

Supplement of

\title{
Temporal rainfall disaggregation using a micro-canonical cascade model: possibilities to improve the autocorrelation
}

\author{
Hannes Müller-Thomy \\ Correspondence to: Hannes Müller-Thomy (mueller-thomy@ hydro.tuwien.ac.at)
}

The copyright of individual parts of the supplement might differ from the CC BY 4.0 License. 


\section{Supplementary Material: Leave-one-out cross validation}

As pointed out in Section 3.1.4 method C and its position-definition increases the number of model parameters. To ensure the model parameter transferability in space a leave-one-out cross-validation was applied. A daily time series was aggregated from an observed $5 \mathrm{~min}$ time series and is referred to as target station. The parameters for the disaggregation were estimated

5 two times, i) from the high-resolution time series of the target station $(T)$ used for the aggregation as well as ii) from the 5 min time series of the closest recording station referred to as neighbouring station $(N)$. The average distance was $\overline{N T}=38.9 \mathrm{~km}$.

The results for the disaggregation are presented in Table S1 and Fig. S1, whereby the results for parameter estimation at the target station are identical with the results presented in Table 4 and Fig. 9. The rainfall characteristics of the disaggregated

10 time series are very similar between both locations $(T$ and $N$ ) for parameter estimation with deviations of less than $5 \%$ for $r E$ and $r A E$. Also the results for the comparison of lag 1, lag 6 and lag 36 autocorrelation are very similar. While for lag 1 the median tends to slightly overestimate the observations for parameters estimated at $T$, parameters estimated at $N$ lead to a slight underestimation. However, for lag $1 r E=0.01$ and $r A E=0.05$ are identical for both locations. Also, the overall representation of the autocorrelation regarding range of the results and tendencies for over- and underestimation is similar

15 between both locations for parameter estimation.

So although method $\mathrm{C}$ is based on a high number of model parameters, their transferability in space is confirmed by this leave-one-out cross validation for the study area.

Table S1: Relative and absolute error of rainfall characteristics between disaggregated and observed time series in dependence of the location used for parameter estimation (mean for 24 stations)

\begin{tabular}{|c|c|c|c|c|c|c|c|c|c|c|c|c|c|}
\hline & & \multicolumn{6}{|c|}{$r E$ [\%] } & \multicolumn{6}{|c|}{$r A E[\%]$} \\
\hline & & $\begin{array}{l}\frac{c}{0} \\
\frac{0}{0} \\
\frac{0}{5} \\
\frac{0}{0} \\
\overline{\bar{d}} \\
0 \\
0 \\
\stackrel{0}{0}\end{array}$ & 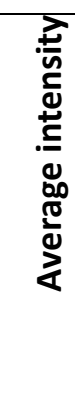 & 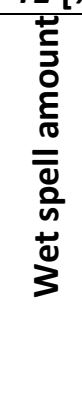 & $\begin{array}{l}\frac{c}{0} \\
\stackrel{0}{0} \\
\frac{0}{3} \\
\frac{0}{0} \\
\overline{\overline{0}} \\
\frac{0}{0} \\
\geq \\
\frac{1}{2}\end{array}$ & 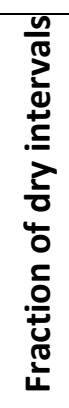 & 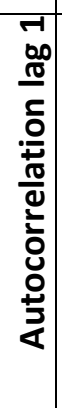 & 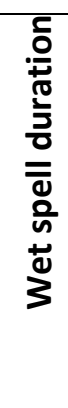 & 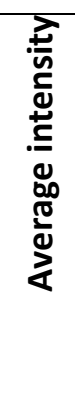 & 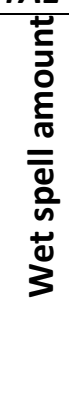 & 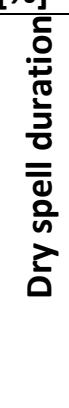 & 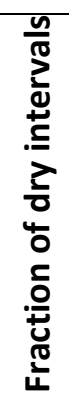 & 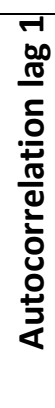 \\
\hline \multirow{2}{*}{ Method C } & & -16 & -35 & -45 & -47 & -3 & 1 & 16 & 35 & 45 & 47 & 3 & 5 \\
\hline & $\mathbf{N}$ & -16 & -32 & -44 & -45 & -3 & 1 & 16 & 32 & 44 & 45 & 3 & 5 \\
\hline
\end{tabular}




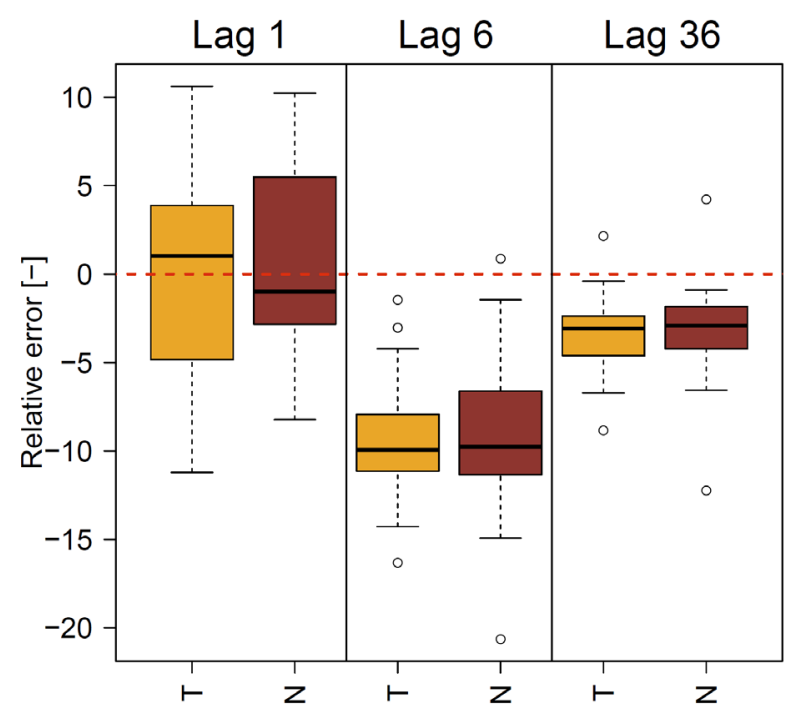

Fig. S1: Deviations of autocorrelation from disaggregated to observed time series for method $\mathrm{C}$ as $r E$ for lags 1,6 and 36 in dependence of the location used for parameter estimation. The red dashed line indicates a $r E=0$ (Std is used as abbreviation for Standard) 